\title{
Study of neutrophil gelatinase-associated lipocalin in patients with cardiovascular shock
}

\author{
Aiyoub Pezeshgi ${ }^{1,2}$, Samad Ghodrati ${ }^{2}$, Mina Kiafar ${ }^{2}$, Kurosh Kamali ${ }^{3}$, Masoud Asadi-Khiavi ${ }^{4,5^{*}}$ \\ ${ }^{1}$ Zanjan Metabolic Disease Research Center, Zanjan University of Medical Sciences, Zanjan, Iran \\ ${ }^{2}$ Department of Internal Medicine, School of Medicine, Zanjan University of Medical Sciences, Zanjan, Iran \\ ${ }^{3}$ Department of Epidemiology, Zanjan University of Medical Sciences, Zanjan, Iran \\ ${ }^{4}$ Zanjan Applied Pharmacology Research Center, Zanjan University of Medical Sciences, Zanjan, Iran \\ ${ }^{5}$ Department of Pharmacotherapy, School of Pharmacy, Zanjan University of Medical Sciences, Zanjan, Iran
}

\section{A R T I C L E I N F O}

\section{Article Type:}

Original

\section{Article History:}

Received: 20 October 2017

Accepted: 10 January 2018

Published online: 18 February 2018

\section{Keywords:}

Neutrophil gelatinase-associated lipocalin, Cardiovascular shock, Acute kidney injury, Hypotension

\begin{abstract}
A B S T R A C T
Introduction: Acute kidney injury (AKI) makes a reversible accumulation of nitrogen products. This waste product is partly determined by serum creatinine level but it is not reliable during hypotension. However, neutrophil gelatinase-associated lipocalin (NGAL), as a new biomarker, shows an obvious increase even at hypotensive status.

Objectives: The presented study was designed to evaluate NGAL as a right biomarker for AKI early diagnosis and consequent appropriate therapies.

Patients and Methods: In this study, 25 healthy individuals and 47 cases out of 60 primarily admitted patients with low blood pressure were evaluated for NGAL level using blood samplings, health documents as well as analysis of questionnaires data. The group's sizes were determined based on AKI and hypotension risk rates. Exclusion and inclusion criteria were firmly considered to avoid major confounding factors.

Results: AKI was found in 20 cases out of 47 hypotensive patients. NGAL levels were about $243.35 \pm 105.74 \mathrm{ng} / \mathrm{dL}($ Mean $\pm \mathrm{SD})$ in AKI and 192.32 $\pm 64.31 \mathrm{ng} / \mathrm{dL}($ Mean $\pm \mathrm{SD})$ in non-AKI hypotensive patients that showed a significant difference $(P=0.037)$ at the first 6 hours. There was no significant difference between hospitalization duration and NGAL level $(P=0.616)$. Conclusion: NGAL is important diagnostic protein in the early stages of AKI while there was no creatinine increasing. On the other hand, NGAL level during early 6 hours of hypotension introduces it as an indicative biomarker of AKI based on provided literature and our presented results.
\end{abstract}

\section{Implication for health policy/practice/research/medical education:}

In the presented study, the diagnostic advantage of neutrophil gelatinase-associated lipocalin (NGAL) was illustrated in AKI. On the other hand, it is concluded that the NGAL is very useful biomarker particularly at the early stages of low blood pressure status in comparison with the routine serum creatinine.

Please cite this paper as: Pezeshgi A, Ghodrati S, Kiafar M, Kamali K, Asadi-Khiavi M. Study of neutrophil gelatinase-associated lipocalin in patients with cardiovascular shock. J Renal Inj Prev. 2018;7(3):144-147. doi: 10.15171/jrip.2018.36.

\section{Introduction}

Acute renal injury refers to a rapid decline in kidney excretory and filtration function. It leads to accumulation of waste products such as creatinine as well as following potassium and phosphate increase (1). More than $30 \%$ of acute kidney injury (AKI) patients show serum creatinine increase by the rate of 0.3 to $0.4 \mathrm{mg} / \mathrm{dL}$. Unfortunately, up to $70 \%$ of mentioned cases illustrate the high rate of mortality in comparison with normal creatinine one (2).
The AKI odds increase by $3 \%$ per $1 \mathrm{~mm} \mathrm{Hg}$ decrease in mean arterial pressure (MAP) less than $80 \mathrm{~mm} \mathrm{Hg}$. Additionally, the risk of AKI enhances two times per hour every 10-mm Hg MAP drop off (3). Several biomarkers were introduced in the case of AKI. One of these markers is serum creatinine that partly indicates nitrogen waste products level but it is not reliable during hypotension. Other biomarker is neutrophil gelatinase-associated lipocalin (NGAL) that showed some advantages rather 
than creatinine. The urinary concentration of NGAL quickly increases during early AKI. Several studies demonstrated that plasma and urine level of NGAL increase during inflammatory processes as well (4). NGAL is discovered in three molecular types; $25-\mathrm{kDa}$ monomer, $45-\mathrm{kDa}$ disulfide-linked homodimer and $135-\mathrm{kDa}$ heterodimer that covalently conjugated with gelatinase [matrix metalloproteinase (MMP)-9] (4-6). These molecules are synthesized by bone marrow and stored within neutrophil granules. Moreover, NGAL mRNA was detected in several organs such as colon, trachea, lung and kidney epithelium (7). Because of NGAL rapid elevation in AKI, it was considered as one of the promising markers for prediction of AKI in Iranian people who need to get appropriate medical services at the right time and as soon as possible.

\section{Objectives}

Presented study was designed to evaluate NGAL as a suitable biomarker for early diagnosis of AKI and consequent proper therapies.

\section{Patients and Methods}

\section{Patients}

A total of 25 healthy volunteers were selected as a group for determining the basal level of NGAL. Additionally, 60 hospitalized patients who had blood pressure drop less than $80 \mathrm{~mm} \mathrm{Hg}$ were primarily considered for comparing accordingly. Inclusion criteria for cases were MAP less than $80 \mathrm{~mm} \mathrm{Hg}$ as well as glomerular filtration rate (GFR) lower than $30 \mathrm{~mL} / \mathrm{min}$ (based on Cockroft-Gault equation). Exclusion criteria for cases were about patients who used nephrotoxic agents like antibiotics, nonsteroidal anti-inflammatory drugs (NSAIDs), angiotensin receptor blockers (ARBs) and angiotensin-convertingenzyme inhibitor (ACE inhibitor) drugs during the week before hypotension attack or patients who administered them during the first 3 days of the hypotension attack. Increased serum bilirubin and death were other exclusion criteria.

\section{Study design}

RIFLE (Risk, Injury, Failure, Loss and ESRD; end stage renal disease) criteria were considered for AKI defining and data gathering. Mentioned 25 healthy volunteers and 60 hospitalized patients were enrolled in the presented survey as a cohort study and blood sampling were done for each group. Creatinine (Cr) and NGAL serum levels were primarily determined at the time of admission and second sampling was performed during first six hours for both groups (during first six hours of blood pressure drop in the case of hospitalized one). Serum creatinine level was also assessed for three consecutive days in the case of hospitalized patients. Serum NGAL level was compared to serum Cr level as a biomarker for AKI. Demographic information such as age, gender, concomitant diseases such as hypertension, diabetes and coronary artery disease was registered in the questionnaires. Subsequently, blood samples $(5 \mathrm{~mL})$ were obtained in the pattern that mentioned previously. The serum level of NGAL was detected by fluorescence-detected immunoassay (ELISA) with the triage meter using Biotech Cristal Day kit.

\section{Ethical issues}

Study related information was delivered to patients and informed consent was provided before using questionnaires. Other information and patients follow up (statues including kidney injury recurrence, potential kidney injury severity, need for dialysis, etc.) were obtained using patients' records and declaration. The research followed the tenets of the Declaration of Helsinki. The Ethics Committee of Zanjan University of Medical Sciences approved this study (ethical code number\# 1-3).

\section{Statistical analysis}

Acquired data were analyzed via paired $t$ test and analysis of variance (ANOVA) following by Bonferroni post-hoc test using SPSS software version 18. The non-parametric tests were used in the case of values without normal distribution. The level less than 0.05 was considered as significant for $P$ value.

Results

\section{Enrolled cases}

As it is mentioned already, a total number of 25 healthy volunteers as well as 60 hospitalized patients were enrolled in study. According to mentioned exclusion criteria, 47 out of 60 patients were evaluated for NGAL level. The mean \pm SD age for healthy volunteers group (12 females and 13 males) was $29.7 \pm 6.8$ years old. This value was about 59.6 \pm 20.9 years in case of patients group ( 19 females and 28 males).

\section{NGAL level values}

Serum creatinine and NGAL mean $( \pm$ SD) level in the healthy volunteers group were $0.9 \pm 0.2 \mathrm{mg} / \mathrm{dL}$ and 246.5 $\pm 84.4 \mathrm{ng} / \mathrm{mL}$ respectively. There was no significant difference between mean values of age and NGAL level in healthy volunteers group, but serum creatinine mean level showed a significant difference between two genders $(P=0.04)$. In the first day, creatinine mean level was 0.9 $\pm 0.1 \mathrm{mg} / \mathrm{dL}$ and was $1.3 \pm 0.9 \mathrm{mg} / \mathrm{dL}$ in the third day that showed an elevated value in comparison with first day. Serum NGAL mean level in this group was about $214 \pm 87.2 \mathrm{ng} / \mathrm{dL}$. The average length of hospitalization was $12.3 \pm 16.3$ days. Unfortunately, thirteen patients expired during hospitalization and no need to use hemodialysis during the study. Creatinine levels of 14 patients showed enhancement up to AKI range. Additionally, 16 patients showed a decline in urine output and 20 patients suffered from AKI based on RIFLE criteria. Mean NGAL level of patients who diagnosed as hypotensive AKI (20 cases) and hypotensive cases without AKI (27 patients) were 243.3 $\pm 105.7 \mathrm{ng} / \mathrm{mL}$ and $192.3 \pm 64.1 \mathrm{ng} / \mathrm{mL}$ respectively that 
showed a significant difference $(P=0.03)$ (Bonferroni post hoc test). There was no significant difference between mean NGAL level of healthy volunteers and AKI as well as non-AKI hypotensive patients $(P=0.89)$. Mean creatinine level of the blood samples in patients group showed significant differences using t-test between second day $(P=0.003)$ and third day $(P=0.001)$ measurements. Additionally, statistical difference between average length of hospitalization period and AKI occurrence was not significant based on mentioned t-test $(P=0.236)$. Chisquare test showed no significant relationship between average length of hospitalization period and mean NGAL level $(P=0.333)$. There was a significant difference between the occurrence of AKI and the RIFLE criteria regarding serum creatinine among first $(P=0.04)$, second $(P<0.001)$ and third days $(P<0.001)$ using t-test. However, such significant differences were not showed about the mean NGAL level $(P=0.062)$. There were significant differences at the second day $(P=0.042)$ and third day $(P=0.04)$ between creatinine and mean NGAL level $(P=0.022)$ in the cases of AKI occurrence and the urinary output except for first day creatinine that showed no significant differences $(P=0.972)$.

\section{Discussion}

Mean creatinine level differences were statistically significant between two genders $(P=0.04)$. This finding is similar to other studies and probably it was about genders with different muscular mass (8). There were significant differences $(P=0.037)$ between mean NGAL levels of hypotensive patients with or without AKI particularly at the early stages of AKI that serum creatinine shows no change. It means that NGAL illustrates a unique property to be considered as a bio-indicator during hypotension as the main etiology of AKI. It was confirmed that AKI occurrence in sepsis was accompanied by high level of NGAL at the admission time (9). It was also showed that critically ill children who affected by SIRS (systemic inflammatory response syndrome) and suspected to severe sepsis have had a significant relation between AKI and NGAL's serum level particularly in first 24 hours of hospitalization (10). There was no significant difference between NGAL level and patient's mortality $(P=0.381)$ in our study. In one study, elevated NGAL level because of malignancy surgery as well as association with postoperation AKI was showed no significant differences between mortality rate and NGAL levels that were as the same of our finding (11). These results are the same as our previous released document in the case of kidney transplantation (12). In other study that handled on 45 patients with cardiogenic shock, it is showed that NGAL level increase at second they can be considered as a mortality predictor for patients with complicated AKI (13). Our findings showed no statically significant differences between serum creatinine and NGAL levels $(P=0.062)$ based on RIFLE criteria. According to RIFLE criteria, urine output values showed significant differences in the case of serum creatinine level at the second $(P=0.042)$ and third $(P=0.04)$ days as well as urine NGAL level $(P=0.022)$ based on our study. This fact was shown in a study on 83 hospitalized patients who admitted in ICU. They measured urine output, NGAL and serum creatinine levels at 24 and 48 hours after hospitalization. They showed a negative relationship between urine NGAL level in severe AKI patients and urine output at the first and second days. However, a direct relation between urine NGAL and serum creatinine at the first day was observed (14). It is illustrated that plasma levels of NGAL at the admission time in post-cardiac surgery ICU is related to average length of hospitalization period in ICU (15). It is against our finding that showed no statically significant difference between NGAL level and average length of hospitalization period. It probably shows less power of our study to judge about relationship between NGAL level and outcomes of AKI. However, variation in outcomes are related to the complexity of subcellular biochemistry and need to understand interactions between genes and use genetic regulatory networks modeling for elucidating molecular bases of mentioned multiple consequences (16).

\section{Conclusion}

The NGAL level increase within the first six hours of hypotension can be considered as an indicator for early AKI during their hospitalization period. Additionally, patients at risk for AKI whose creatinine level has not elevated yet can be detected using NGAL level.

\section{Limitations of study}

According to the variety of NGAL level measurements and limited size of healthy volunteers group, further studies with the large size of healthy volunteers is essential.

\section{Authors' contribution}

AP; scientific writing, principal co-author, coordinator. SG; second co-author, help in scientific writing. MK; patients detecting. KK; statistical analysis. MAK; principal author, corresponding author.

\section{Conflicts of interest}

The authors declare no conflict of interest.

\section{Ethical considerations}

Ethical issues (including plagiarism, misconduct, data fabrication, falsification, double publication or submission, redundancy) have been completely observed by the authors.

\section{Funding/Support}

This article is extracted from residential thesis of Mina Kiafar (Thesis number: A-11-340-1). This study was partly supported by a grant from Zanjan University of Medical Sciences (\# a-12-635-1, 2015). 


\section{References}

1. Bellomo R, A Kellum J, Ronco C. Acute kidney injury. The Lancet. 2012;380:756-66. doi: 10.1016/S01406736(11)61454-2.

2. Chertow GM, Burdick E, Honour M, Bonventre JV, Bates DW. Acute kidney injury, mortality, length of stay, and costs in hospitalized patients. J Am Soc Nephrol. 2005;16:336570. doi: 10.1681/ASN.2004090740

3. Lehman L, Saeed M, Moody G, Mark R. Hypotension as a risk factor for acute kidney injury in ICU patients. Comput Cardiol. 2010;37:1095-1098.

4. Martensson J. Bellomo R. The rise and fall of NGAL in acute kidney injury. Blood Purif. 2014;37:304-10. doi: $10.1159 / 000364937$

5. De Geus HR, Bakker J, Lesaffre EM, Le Noble JL. Neutrophil gelatinase-associated lipocalin at ICU admission predicts for acute kidney injury in adult patients. Am J RespirCrit Care Med. 2011;183:907-14. doi: 10.1164/rccm.2009081214OC.

6. Cowland JB, Borregaard N. Molecular characterization and pattern of tissue expression of the gene for neutrophil gelatinase-associated lipocalin from humans. Genomics: 1997;45:17-23. doi: 10.1006/geno.1997.4896

7. Mishra J, Ma Q, Prada A, Mitsnefes M, Zahedi K, Yang J, et al. Identification of neutrophil gelatinase-associated lipocalin as a novel early urinary biomarker for ischemic renal injury. J Am Soc Nephrol. 2003;14:2534-43.

8. Verma M, Khadapkar R, Sahu PS, Das BR. Comparing agewise reference intervals for serum Creatinine concentration in a "Reality check" of the recommended cut-off. Indian J Clin Biochem. 2006;21:90-4. doi: 10.1007/BF02912919.

9. Vanmassenhove J, Glorieux G, Lameire N, Hoste E, Dhondt A, Vanholder R, et al. Influence of severity of illness on neutrophil gelatinase-associated lipocalin performance as a marker of acute kidney injury: a prospective cohort study of patients with sepsis. BMC Nephrol. 2015;16:18. doi: 10.1186/s12882-015-0003-y.

10. Wheeler DS, Devarajan P, Ma Q, Harmon K, Monaco M, Cvijanovich N, et al. Serum neutrophil gelatinase-associated lipocalin (NGAL) as a marker of acute kidney injury in critically ill children with septic shock. Crit Care Med. 2008;36:1297-303. doi: 10.1097/CCM.0b013e318169245a.

11. Delfino Duarte PA, Fumagalli AC, Wandeur V, Becker D. Urinary neutrophil gelatinase-associated lipocalin in critically ill surgical cancer patients. Indian J Crit Care Med. 2015;19:251-6. doi: 10.4103/0972-5229.156459.

12. Pezeshgi A, Abedi Azar S, Ghasemi H, Kamali K, Esmaeilzadeh A, Hajsalimi B, et al. Role of plasma neutrophil gelatinase-associated lipocalin as an emerging biomarker of acute renal failure following kidney transplantation and its correlation with plasma creatinine. J Renal Inj Prev. 2016;5:98-103. doi: 10.15171/jrip.2016.21.

13. Fouda M, Sherif $H$, Shehata M, Ibrahim A. Early expression of urinary neutrophil gelatinase-associated lipocalin biomarker predicts acute kidney injury complicating circulatory shock. Egypt J Crit Care Med. 2013;1:79-86.

14. Watanabe M, Fulan e Silva G, Dezoti da Fonseca C, Vattimo MFF. Urinary NGAL in patients with and without acute kidney injury in a cardiology intensive care unit. Rev Bras Ter Intensiva. 2014;26:347-54. doi: 10.5935/0103507X.20140053.

15. Mao H, Katz N, Ariyanon W, Blanca-Martos L, Adýbelli Z, Giuliani A, et al. Cardiac surgery-associated acute kidney injury. Cardiorenal Med. 2013;3:178-99. doi: $10.1159 / 000353134$.

16. Yaghoobi H, Haghipour S, Hamzeiy H, Asadi-Khiavi M. A review of modeling techniques for genetic regulatory networks. J Med Signals Sens. 2012;2:61-70.

Copyright (c) 2018 The Author(s); Published by Nickan Research Institute. This is an open-access article distributed under the terms of the Creative Commons Attribution License (http://creativecommons.org/licenses/by/4.0), which permits unrestricted use, distribution, and reproduction in any medium, provided the original work is properly cited. 